\title{
Dying to Learn: The Supply and Use of Companion Animals in U.S. Colleges and Universities*
}

\author{
Laura Ducceschi, Nicole Green and Crystal Miller-Spiegel \\ Animalearn, Jenkintown, PA, USA
}

\begin{abstract}
Summary
Americans consider dogs and cats as household pets, but many are harmed and killed for teaching and training purposes, despite the availability of alternatives. A review of 92 U.S. public college and university Institutional Animal Care and Use Committee (IACUC) 2005-2007 records indicates that 52\% are using live and dead dogs and cats, and 26\% are using live dogs and cats in harmful teaching exercises in undergraduate life science, veterinary, and medical education. In specific cases, IACUCs are failing to minimize animal use and suffering in education as required by the Animal Welfare Act (AWA). Sources of dogs and cats for education include Class A and Class B dealers, and United States Department of Agriculture (USDA) 2005-2007 inspections reveal repeated violations and inhumane treatment. Regardless, dealers continue to sell thousands of dogs and cats, many whom were former pets, annually to universities for use in education. A growing number of universities, however, are changing their policies and replacing harmful animal use with pedagogically sound alternatives.
\end{abstract}

Keywords: canine, education, feline, training, university, dissection, veterinary

\section{Introduction}

Since Animalearn's inception in 1990, educators and students have inquired as to the origins of animals being used for educational purposes in the United States. They are often surprised to learn that dogs and cats not only are used but are also harmed and even killed for educational purposes in undergraduate, veterinary, and medical education. Many question where colleges and universities obtain these animals. Publicly available information to answer these questions was lacking. In response, Animalearn investigated the acquisition and use of dogs and cats in teaching and training labs in public colleges and universities across the U.S., as well as the replacement of the use of these animals with humane alternatives in undergraduate, veterinary, and medical education.

\section{Dogs and cats under the AWA}

Federal protection for dogs and cats was first enacted in 1966, when the Animal Welfare Act (AWA) was introduced in large part to address concerns regarding the use of illegally obtained cats and dogs, many of whom were former pets, in experimentation ${ }^{1}$. Since its inception, the AWA has been amended several times, with some intentions of the 1985 amendments aimed at decreasing animal suffering by encouraging the use of alternatives $^{2}$. Under the AWA, cats and dogs, as well as other animals, are afforded basic legal protections related to their care and use. To further this purpose, Congress provided that investigators who wish to use animals for research or teaching purposes must first consider alternatives to any procedure likely to produce pain or distress in an animal and eliminate the unnecessary duplication of experiments on animals ${ }^{3}$. The AWA establishes the Institutional Animal Care and Use Committee (IACUC) as a self-regulating entity that must exist to oversee and evaluate all aspects of an institution's animal care and use program, if they use animals covered under the AWA for research or instructional purposes.

Despite the intent of the AWA, companion animals and other animals continue to suffer unnecessarily to provide educational experiences for undergraduate, graduate, veterinary, and medical students at some colleges and universities. Recently, how-

\footnotetext{
* this article was presented as a poster at the $7^{\text {th }}$ World Congress Rome 2009; the full report of this article is available at http://www.dyingtolearn.org

1 Animal Welfare Act. 7 U.S.C. $\S 2131 \S 2132(\mathrm{e})$.

2 Animal Welfare Act. 131 Cong. Rec. 29,155 (1985).

3 Animal Welfare Act. 7 U.S.C. $\S 2143$ (a)(3)(B) and id. § 2131 (c) (3).
} 
ever, many schools have been moving towards offering students alternatives, due in large part to student advocacy efforts and the opportunities presented by advances in technology (Mangan, 2007).

\section{Animals, materials and methods}

To estimate the use of dogs and cats in higher education in the U.S., Animalearn queried public colleges and universities located in a sample of 24 states (175 institutions). Many of these schools also have veterinary and medical schools. The sample of 175 locations is both broad and diverse, and the procurement and use of dogs and cats for educational purposes in other colleges and universities not included in this sample would likely be similar.

Data were acquired via three methods:

1. IACUC public records 2005-2007

Information on the number and type of dogs and cats purchased or acquired for teaching purposes.

2. USDA inspection reports and license renewal applications Freedom of Information Act (FOIA) requests to the USDA for those licensed Class A and Class B animal dealers (Random Source and Biological Supply Companies) selling to colleges and universities in the sample to obtain information on sales of dogs and cats and records of regulatory violations.

3. Survey of university biology departments 150 biology departments from 175 institutions regarding their use of live and dead dogs and cats, how they are used, and whether students are permitted to use alternatives.

\section{Results}

1. Universities are harming and killing dogs and cats to fulfill educational objectives that can be met by alternatives.

These teaching exercises include terminal surgery labs at veterinary and medical schools in which dogs are killed following the procedure; clinical skills training labs for veterinary students, which involve euthanizing live dogs or cats in order to teach specific skills to students; and animal dissection, a practice used to teach anatomy and physiology. Many animals are killed specifically for use in education, even though viable alternatives are being implemented successfully at other universities.

2. Of 92 universities reviewed from 2005-2007, the records indicate that $52 \%$ are using live or dead dogs and cats and $26 \%$ are using live dogs and cats.

3. Of 150 university biology departments surveyed in 2008 (20\% response rate), $63 \%$ are using dead cats to teach anatomy and physiology.

A separate survey of university biology departments indicates that more than half of the respondents are using cat cadavers to teach anatomy/physiology in undergraduate classrooms. Cats, and occasionally dogs, continue to be used for dissection in comparative anatomy classes or as surrogates for humans in human anatomy.

\section{Universities are acquiring dogs and cats from inhumane sources.}

Suppliers of dogs and cats used in education include Class A Dealers, or animal breeders; Class B Dealers (Random source) which purchase and re-sell live and/or dead animals from auction sales, other dealers, pounds and shelters, and private persons; Class B Dealers (Biological supply companies), which provide live and dead animals to classrooms and laboratories; and Pound Seizure, or the sale or release of cats and dogs from a pound or shelter to a research, testing, or educational facility (Tab. 1).

Many Class A and Class B Dealers have consistent patterns of AWA violations, including falsifying records and providing inadequate animal care, resulting in animal suffering and distress. In addition, universities are going directly to animal pounds and shelters to acquire animals through pound seizure (Tab. 1).

\section{IACUCs are failing to provide effective oversight.}

In-depth analysis of approved animal use protocols (AUPs) from selected university IACUCs indicates disregard of valid database search procedures for alternatives. Specifically, AUPs revealed patterns of "canned" searches, incorrect and irrelevant search terms, and a limited range of database use, as well as misrepresentation of results.

\section{A growing number of universities are changing their policies and replacing harmful animal use with pedagogically sound alternatives.}

Practices and policies include Educational Memorial Programs (EMPs) or Willed Body Donation programs; Shelter medicine programs; Surgical simulation; Virtual dissection; Student Choice policies; and "No Random Source Animals" policies.

For example, The College of Veterinary Medicine (CVM) at the University of Georgia (UGA) is instituting a Shelter medicine program, and a DVD/digital media library of non-terminal (spay/neuter) surgical procedures (both partially funded by Animalearn). UGA CVM is also initiating a small animal Educational Memorial Program (EMP). The end goal of these policies is the elimination of terminal surgeries in veterinary curriculum.

\section{Discussion: Recommended best practices for replacing the harmful use of dogs and cats in teaching and training labs in life science, veterinary, and medical education.}

\section{Create curricula that identify or utilize alternatives as the default procedure in life science, veterinary, and medical education.}

The harmful use of animals is unnecessary due to the variety of pedagogically sound alternatives available to replace animal experiments in education. Alternatives can be used to teach anatomy, simulate biological functions, and practice clinical 
Tab. 1: Sources of live dogs and cats used for higher education, 2005-2007

\begin{tabular}{|c|c|c|c|c|}
\hline College/University & $\begin{array}{l}\text { Class A } \\
\text { Dealer }\end{array}$ & $\begin{array}{l}\text { Class B } \\
\text { Dealer }\end{array}$ & $\begin{array}{l}\text { Pound } \\
\text { Seizure' }^{1}\end{array}$ & $\begin{array}{l}\text { Other } \\
\text { Sources }{ }^{2}\end{array}$ \\
\hline Auburn University & & & & $X$ \\
\hline Colorado State University, Fort Collins & $x$ & & & \\
\hline Iowa State University & & & $X^{3}$ & $x$ \\
\hline Michigan State University, East Lansing & $x$ & $x$ & $x$ & $X$ \\
\hline Oakland University, Rochester & $x$ & & & \\
\hline Ohio State University & & $x$ & & \\
\hline Oklahoma State University & $x$ & $x$ & & \\
\hline Purdue University & & $x$ & & $x$ \\
\hline Texas A\&M University, College Station & $x$ & & $x$ & $x$ \\
\hline University Of California, Davis & & & & $x$ \\
\hline University of California, Santa Barbara & $x$ & & & \\
\hline University of Cincinnati & $x$ & & & \\
\hline University of Connecticut, Storrs & $x$ & & & \\
\hline University of Florida, Gainesville & $x$ & $x$ & & \\
\hline University of Georgia, Athens & & $x$ & $x$ & \\
\hline University of Illinois, Chicago & & $x$ & & \\
\hline University of Michigan, Ann Arbor & $x$ & $x$ & & \\
\hline University of Minnesota, St. Paul & $x$ & $x$ & $x^{3}$ & \\
\hline University of North Carolina, Chapel Hill & $x$ & & & \\
\hline University of Oklahoma, Health Sciences Center & & $x$ & & \\
\hline University of Texas, Dallas ${ }^{4}$ & $x$ & & & $X$ \\
\hline University of Texas, Southwest Medical Center & $x$ & & & $x$ \\
\hline University of Washington, Seattle & $x$ & & & $x$ \\
\hline University of Wisconsin, Madison & $x$ & & & $x$ \\
\hline
\end{tabular}

Dealer(s) in category are cited for Animal Welfare Act violations.

${ }^{1}$ Pound seizure column includes live animals only.

${ }^{2}$ Other sources include other university departments, other colleges and universities, and donations.

${ }^{3}$ These animals may have been used for beneficial spay/neuter surgeries and then returned to shelter.

${ }^{4}$ Stopped using cats in 2008 . No dogs used. 
and surgical skills. They allow students to perform tasks at their own pace, repeating if necessary until the students master the material or the techniques being taught. Often costing less over the long-term than using animals, alternatives are humane, educationally effective, economical, and save countless animal lives. They are available free on loan through alternatives loan programs like Animalearn's The Science Bank.

Alternatives that can replace the harmful use of dogs and cats in teaching and training labs include: manikins (e.g., Rescue Critters' Critical Care Jerry, Paws 2 Claws Spay Manikin; SimPooch); ethically sourced plastinated specimens; virtual dissection; CDRoms (e.g., BioLab Cat, Canine Osteology); software for human anatomy and physiology (since dogs and cats are often used as surrogates for human anatomy); ethically sourced animal cadavers (accessible through willed body donation); human cadavers (accessible through willed body donation); virtual reality surgical simulation (e.g., The Ohio State University's School of Veterinary Medicine's virtual surgical simulation) (Stredney et al., 2006); and Live Surgery Simulation (e.g., Dr. Emad Aboud's Advanced Surgical Simulator) (Aboud et al., 2002).

\section{Institute educational programs that are beneficial to animals.}

Educational Memorial Programs (EMPs) or Willed Body Donation programs offer client-donated cadavers and can be established in order to provide ethically sourced animals. Donated animals offer case-based learning opportunities, where students may receive a complete medical history, as they might with an actual animal patient. Students can rotate between stations, increasing their knowledge of pathological conditions and surrounding anatomy (Kumar et al., 2001).

Shelter Medicine programs allow veterinary schools to eliminate terminal surgery labs, enabling students to obtain hands-on experience performing beneficial surgeries, such as spays and neuters. Collaborations between veterinary schools and animal shelters provide students with crucial opportunities for necessary skills acquisition, particularly for teaching medical and surgical skills, post-operative recovery, handling live animals, and working with live tissue. In addition, these programs provide an important service for local communities, as they are vital in helping to reduce overpopulation.

\section{Develop institutional policies that promote humane practices.}

Establishing a formal student choice policy allows students to establish their right to choose an alternative to using animals in education. A formal, written, student choice policy can be either university-wide or departmental. Animalearn works with college and university students to help them navigate the process of successfully establishing a policy that will allow students to choose an alternative to dissection and laboratory experiments (Ducceschi et al., 2007).

In addition to helping to pass student choice policies, students can foster other policies that can help eliminate the harmful use of animals in education at their university. One example is a
"No Random Source Animals" policy, which explicitly prohibits the acquisition of animals from Class B Dealers (Random source). These policies promote a willingness on the part of a university to avoid contributing to the inhumane treatment that animals often encounter when acquired, housed, and transported by these dealers. Iowa State University (ISU) has instituted a formal "No Random Source Animals" policy (IACUC, undated). To promote humane treatment of dogs, cats, and other animals, however, it is critical that universities enacting such a policy seek out humane sources of animals rather than other animal dealers (e.g. Class A) or pound seizure.

\section{Provide educators with training opportunities for \\ identifying and using appropriate alternatives.}

Federal resources (e.g. USDA's Animal Welfare Information Center) exist for training on the identification and enhancement of alternatives use at the college and university level. There are many organizations (e.g. Animalearn) and agencies that can assist faculty and administrators in locating effective alternatives to using dogs, cats, and other animals in teaching labs.

The Animal Welfare Information Center (AWIC) was established to help research institutions comply with AWA regulations, including a thorough literature search for alternatives to animal use prior to initiating such a procedure. AWIC provides information on peer reviewed studies to assist in locating viable alternatives; assistance with search terminology for conducting the most effective searches; a list of viable alternatives to using animals in medical and veterinary education; and training workshops for university IACUC committee members (Animal Welfare Information Center, 2008).

Animalearn's The Science Bank offers detailed solutions for undergraduate, medical, and veterinary education that can help colleges and universities to eliminate the harmful use of animals from their curricula without sacrificing educational quality. The Science Bank is the largest free loan program in the U.S. for alternatives to animal use, providing more than 450 alternatives to dissection and vivisection, including CD-ROMs, DVDs, realistic models, manikins, and surgical simulators, available in multiple quantities for entire classrooms (Animalearn, undated).

\section{Eliminate inhumane sources of animals used in education.}

Animalearn recommends an immediate prohibition on pound seizure at the federal level. These animals can be replaced with humane alternatives, such as client-donated cat and dog cadavers or beneficial surgeries. Use of these alternatives does not shake the trust that people expect to have in animal shelters but rather allows people to feel comfortable that, if they do have to bring a pet to a shelter, their pet will not end up in a laboratory and used in experiments.

Random source animals should not be used in education. Recommendations include a prohibition on acquiring animals from Class B Dealers (Random source), animal shelters/pounds, or international pounds. This prohibition should be part of federal 
law and state law, as well as included in institutional policies. USDA should exercise its authority by immediately eliminating of Class B Dealer licenses at the federal level.

Biological Supply Companies should not be permitted to obtain animals from U.S. pounds and shelters, nor should they be permitted to transport animals across international borders for sale in the U.S. The USDA should consider them high-risk and inspect them more frequently.

Animalearn recommends that any animals used for educational purposes be ethically-sourced and used in procedures beneficial or therapeutic to the animal. In addition, animals should not be bred specifically for educational use, since alternatives are available for replacement.

\section{Authors Note}

Oct. 2010:This is a very dynamic area, and there are indications that some of the institutions named may have changed their practices since 2007 reporting.

\section{References}

Aboud, E., Al-Mefty, O. and Yasargil, M. G. (2002). New laboratory model for neurosurgical training that simulates live surgery. J. Neurosurg. 97, 1367-1372.

Animal Welfare Information Center. Workshops. (2008). United States Department of Agriculture (USDA). http://awic. nal.usda.gov/nal_display/index.php?info_center $=3 \&$ tax level=1\&tax_subject=188.")
Animalearn (undated). http://www.animalearn.org

Ducceschi, L., Hart, L. A. and Green, N. (2007). Guidelines for the development of student choice policies regarding dissection in colleges and universities: An ethnographic analysis of faculty and student concerns. AATEX 14, Proceedings from the $6^{\text {th }}$ World Congress on Alternatives \& Animal Use in the Life Sciences 4, 273-276.

IACUC - University Policies (undated). Purchase of dogs and cats from USDA Class B Licensees. Iowa State University. http://www.compliance.iastate.edu/ComplianceWeb/coacUniversityLaw.aspx.html\#teaching

Kumar A. et al. (2001). Client donation program for acquiring dogs and cats to teach veterinary gross anatomy. J. Vet. Med. Educ. 28, 73-77.

Mangan, K. (2007). Medical schools stop using dogs and pigs in teaching. Chron. High Educ. 54(7), A12.

Stredney, D. et al. (2006). Simulation technology in veterinary education, research and surgical training. Proceedings 2006 ACVS Veterinary Symposium.

\section{Correspondence to}

Animalearn

801 Old York Road

Suite 204

Jenkintown, PA 19046

USA

e-mail: LDucceschi@animalearn.org 